\title{
Permeance Based Modeling of Magnetic Hysteresis with Inclusion of Eddy Current Effect
}

M. Luo, D. Dujic, and J. Allmeling

This material is posted here with permission of the IEEE. Such permission of the IEEE does not in any way imply IEEE endorsement of any of EPFL's products or services. Internal or personal use of this material is permitted. However, permission to reprint / republish this material for advertising or promotional purposes or for creating new collective works for resale or redistribution must be obtained from the IEEE by writing to pubs-permissions@ieee. org. By choosing to view this document, you agree to all provisions of the copyright laws protecting it. 


\section{Permeance Based Modeling of Magnetic Hysteresis with Inclusion of Eddy Current Effect}

\author{
Min Luo and Drazen Dujic \\ Power Electronics Laboratory \\ École Polytechnique Fédérale de Lausanne (EPFL) \\ Lausanne CH-1015, Switzerland \\ min.luo@epfl.ch,drazen.dujic@epfl.ch
}

\author{
Jost Allmeling \\ Plexim GmbH \\ Zürich CH-8005, Switzerland \\ allmeling@plexim.com
}

\begin{abstract}
Magnetic hysteresis together with eddy current effects are typically present in metal based core materials and contribute significantly to the nonlinearity and power loss of the magnetic components operating in power electronic converters. In order to investigate their influence on the system's behavior in time domain, model which is accurate and simple enough to be integrated into circuit simulation environment, is desired. This work proposes a modeling's approach using permeancecapacitance based magnetic circuit, which combines the hysteresis and eddy current effect of the magnetic components in system-level time domain simulation.
\end{abstract}

Index items-hysteresis, eddy current, permeancecapacitance, magnetic circuit, system-level simulation

\section{INTRODUCTION}

Magnetic components using metal based core materials are commonly applied in power electronic converter systems. The magnetic hysteresis of the core material together with eddy current effect contributes directly to the nonlinearity and power loss of the magnetic component during operation. In order to investigate their influence on the system behavior, so as to adapt the control algorithm and component selection of the rest part of the power converter, model which is accurate and simple enough to be integrated into system-level circuit simulation environment, is desired.

Electrical equivalent circuit model has been commonly used for system-level simulations. The iron core is usually represented by the Cauer-type equivalent circuits as demonstrated in Fig. 1 [1]. The inductors account for the magnetic flux channel while the resistors for the eddy current loss. Using the electrical equivalent circuit, authors of [2] were able to reproduce the analytical form of the core laminate's frequency response, with the material nonlinearity neglected. In the work of [3] and [4] the authors have taken into account the material nonlinearity and the method of [4] has been extended to a multi-winding structure in [5]. There the material characteristic is represented by a single-line nonlinear B-H curve, which does not cover the power loss from the frequency-independent hysteresis effect of the real magnetic materials. The authors of [6] and [7] have included the magnetic hysteresis into the circuit model, however only simple core geometry (toroidal core) was considered, which is not generalised to complex core structures.

Magnetic circuits based on permeance-capacitance analogy introduced by [8] are able to represent complex core geometry intuitively, and have been proved to be practical for system-level circuit simulation in the work

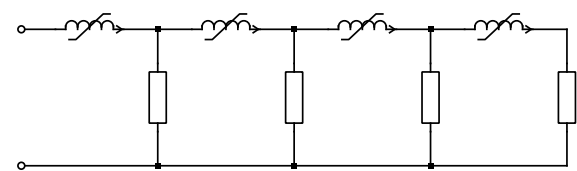

Fig. 1: Cauer equivalent circuits for iron cores. 
of [9], [10] and [11]. The magnetic hysteresis of soft material can be accurately captured via incorporating Preisach model [12]. Thanks to its gyrator structure to interface the electrical part of the model, the magnetic circuit can be simulated in its original form directly. Based on the permeance-capacitance magnetic circuit, the author of [13] has proposed a ladder structure for eddy current effect, however the core material nonlinearity was not taken into account. This work proposes a core model for time domain system-level simulation, which captures the magnetic hysteresis of the core material together with eddy current effect. The model validity is verified experimentally under excitation of different amplitudes and frequencies.

\section{Modeling}

Laminated Silicon Steel ( $\mathrm{SiFe}$ ) material is taken as study case, which has been widely used to construct iron cores like that of the three-phase transformer shown in Fig. 2a. The modeling's approach is introduced using the

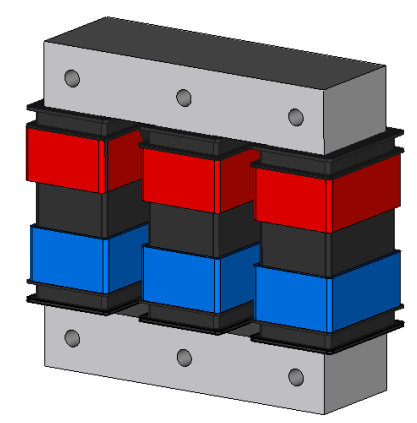

(a)

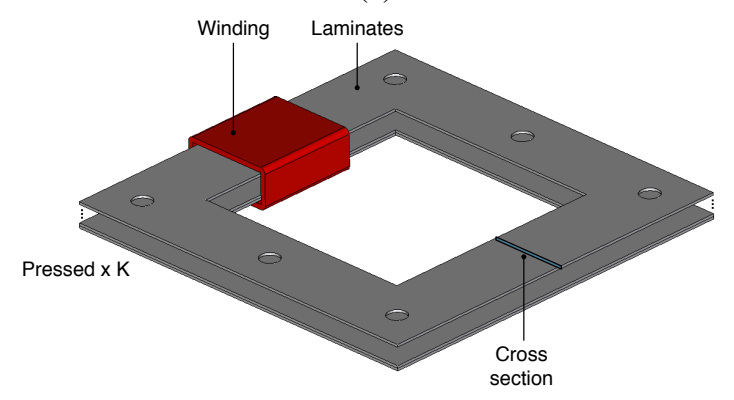

(b)

Fig. 2: (a) Three-phase transformer using laminated silicon steel; (b) Magnetic structure taken as example for demonstration with pressed laminates and one winding.
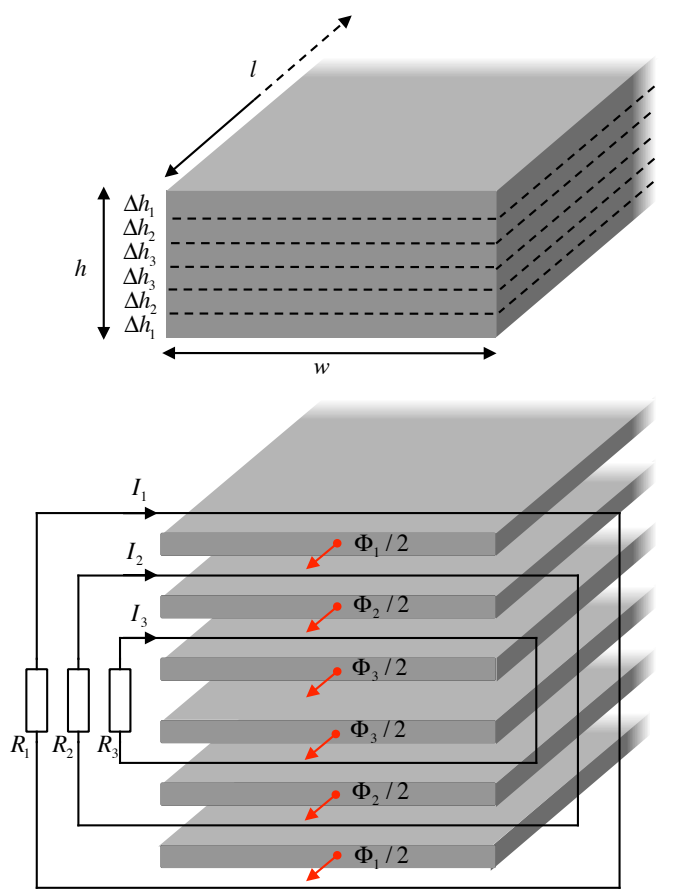

(a)

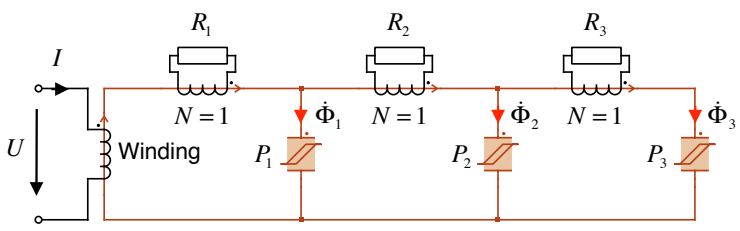

(b)

Fig. 3: (a) Cross section of one single laminate virtually divided into non-conductive sub-laminates and resistor loops; (b) Magnetic circuit of one single laminate with three permeances and resistor loops.

magnetic structure demonstrated in Fig. $2 b$ as example. The iron core is composed of $K$ identical square laminates which are pressed together and electrically isolated from each other. The cross section area of each laminate is $A$ and the equivalent magnetic path length (centre line) is $l$. An electrical winding is bundled on one side of the core.

In order to derive the circuit model, the cross section of one laminate shown in Fig. 3a is virtually divided into several sub-laminates. In this paper, considering the thickness of the laminate we were using, for the modeling purposes six sub-laminates were providing good bal- 
ance between complexity and accuracy. In linear case, [3] has explored that the error of equivalent impedance with six sub-lamination is under $15 \%$. It is assumed that the sub-laminates are non-conductive with magnetic fluxes $\Phi_{1} / 2 \sim \Phi_{3} / 2$ pointing perpendicular to the cross section, while virtual conductive foils are placed surrounding the sub-laminates representing the conductivity of the core material, which can be modelled as short-circuit resistor loops $\left(R_{1} \sim R_{3}\right)$. If only one laminate is present $(K=1)$, the magnetic circuit model can be constructed as shown in Fig. 3b. Each of the three permeances $P_{1} \sim P_{3}$ represent one pair of the non-conductive sub-laminates, through which the fluxes $\Phi_{1} \sim \Phi_{3}$ are flowing. $R_{1} \sim R_{3}$ in the electrical circuit correspond to the virtual resistor loops in Fig. 3a, which are connected to the magnetic circuit via winding block (with turns number $N=1$ ) using gyrator structure. The winding block on the very left hand side of Fig. 3b represents the electrical winding in reality.

Geometrically one can assume that all sub-laminates have the same thickness. However recognising that the flux becomes confined to an increasingly thinner layer near the lamination surface as the frequency increases, nonuniform division proposed by the authors of [3] is adopted in this work. The thickness of the sub-laminates is doubled progressively from the surface to the centre of the lamination, that is:

$$
\Delta h_{k-1}=2 \cdot \Delta h_{k}
$$

while the summation of all $h_{k}$ should be equal to the thickness $h$ of the original lamination.

$$
\sum \Delta h_{k}=h
$$

Following the approach proposed in the work of [12], the nonlinear permeances depending on field strength $H$ are provided as the summation of an irreversible component $P_{k}^{i r r}$ and a reversible component $P_{k}^{r e v}$, scaled by the geometrical parameters of the individual sublaminates ( $\Delta h_{k}, w$ and $l$ in Fig. 3a).

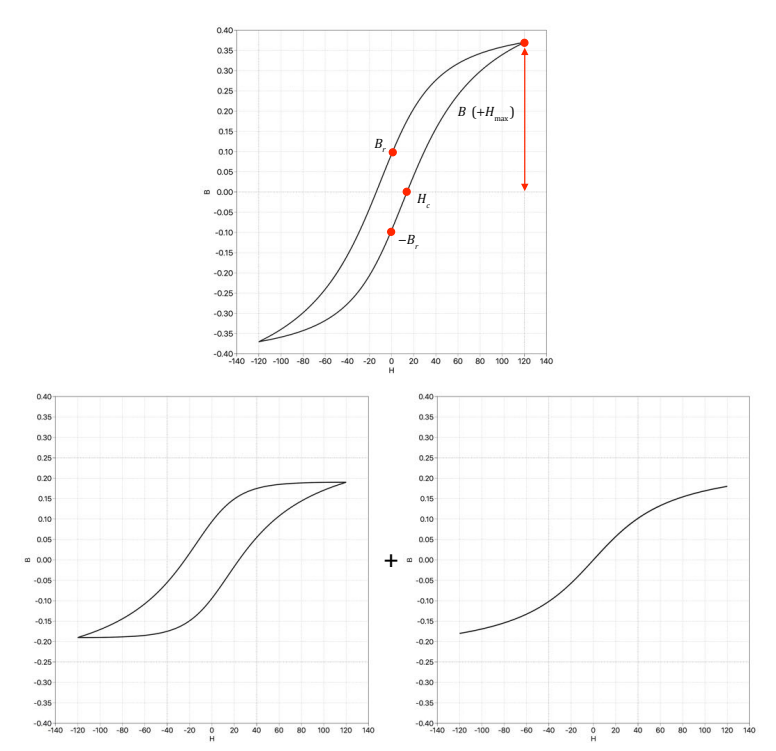

Fig. 4: B-H characteristic of the permeance block as summation of irreversible- and reversible component.

$P_{k}(H)=P_{k}^{i r r}+P_{k}^{r e v}=\left(\mu^{i r r}+\mu^{r e v}\right) \cdot \frac{2 \Delta h_{k} w}{l}$

Please note that the factor 2 in equation indicate the fact that the permeance in each section of the magnetic circuit includes two sub-laminates. The irreversible permeability $\mu^{i r r}(H)$ is realised using classical Preisach model, for which the formulation introduced by [14] is taken. The irreversible $\mu^{r e v}(H)$ is modelled by the inverse trigonometric (arctan) function proposed by [15]. The resulted $\mathrm{B}-\mathrm{H}$ characteristic is the summation of contributions from the irreversible- and reversible component on the $B$ axis, as demonstrated in Fig. 4. The parameters of the permeance model are identified to fit an experimentally measured limiting B-H loop, the field strength range $\left[-H_{\max }, H_{\max }\right]$ of which should be sufficiently large to cover the operation conditions in concern, following the procedure proposed by [12]. Please note that this B-H loop must be obtained at low frequency, where the frequency-dependent eddy current effect has negligible influence on the B-H characteristic.

The resistors $R_{1} \sim R_{3}$ are calculated making use of the sub-laminates' geometry, which are given by: 


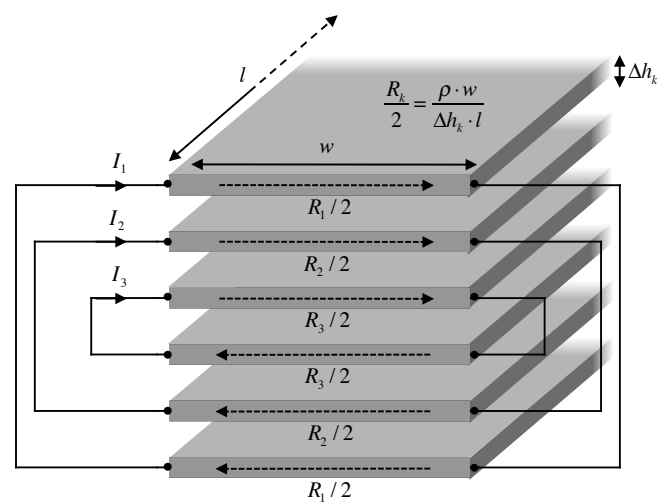

Fig. 5: Equivalent resistance of the sub-laminates

$$
R_{k}=\frac{2 \cdot \rho \cdot w}{\Delta h_{k} \cdot l}
$$

where $\rho$ is the material's resistivity, which can be obtained from the datasheet from the material manufacturer. Please note that equation (4) essentially calculates the equivalent resistance along the horizontal direction using the sub-laminates' geometry but non-zero conductivity, and the factor 2 indicates that the two sub-laminates in the same section are in series connection, as demonstrated in Fig. 5.

If more than one laminates are pressed together, the circuit model becomes parallel connection of $K$ times the circuit shown in Fig. 3b. Merging the parallel items together yields the same structure in Fig. 3b, while the thickness $\Delta h_{k}$ in equation (II) and the resistance $R_{k}$ should be multiplied by $K$.

\section{EXPERIMENTAL VALIDATION}

For validation of the proposed modelling's approach, a test bench based on the approach introduced in [16] has been established to measure the B-H loops, as shown in Fig. 6. The test setup is composed of a RF power amplifier (bandwidth $2 \mathrm{MHz}$ ) to generate arbitrary voltage waveform, as well as a control unit (PLECS RT-Box) to provide reference signal and measurement data access. 15 pieces of square laminates made of silicon steel M330-35 are pressed together creating the core sample for the study. One primary- and one secondary winding

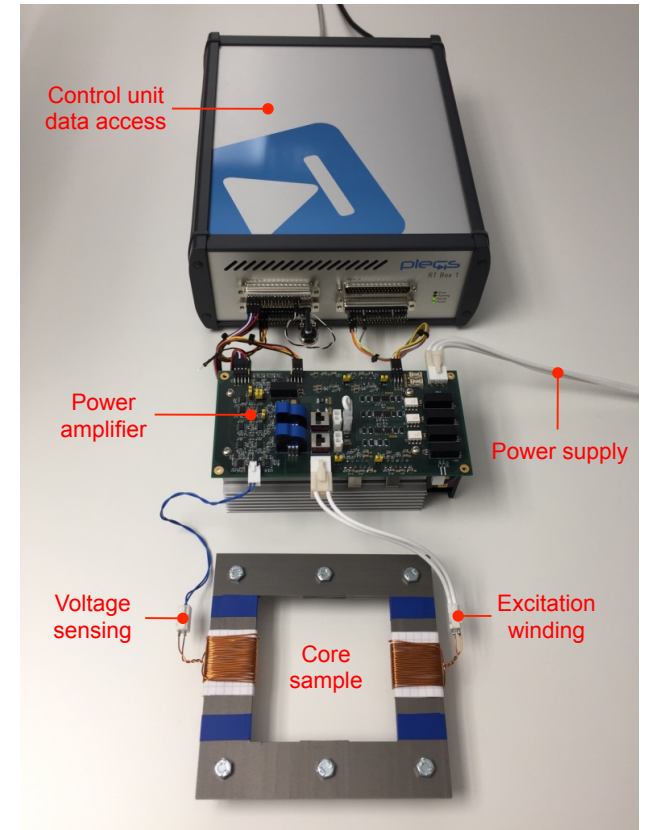

(a)

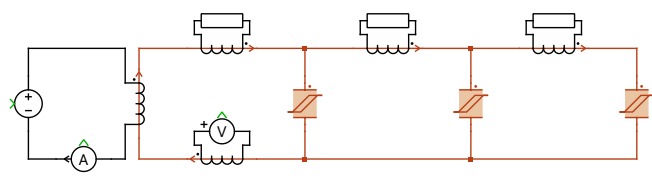

(b)

Fig. 6: Verification setup with the core sample, power amplifier and control unit (a) Realised hardware; (b) Schematic and simulation model.

with the same turns number $N_{1}=N_{2}=30$ are installed on the core sample. The primary winding is supplied by the amplifier and the current is measured to obtain the field strength $H$. The secondary winding is left open and the voltage of which is measured and integrated to obtain the flux density $B$. As the temperature dependency is not the scope of this work, all tests have been carried out under room temperature of $25^{\circ} \mathrm{C}$.

For parameter identification of the classical Preisach model in the permeance blocks, one limiting B-H loop with the peak field strength $H_{\max }=100 \mathrm{~A} / \mathrm{m}$ under $25 \mathrm{~Hz}$ sinusoidal excitation is measured. The parameters of the nonlinear permeances are determined to fit the $25 \mathrm{~Hz}$ B-H loop following the procedure introduced by [12]. After that the resistance values $R_{k}$ in Fig. $3 \mathrm{~b}$ are 


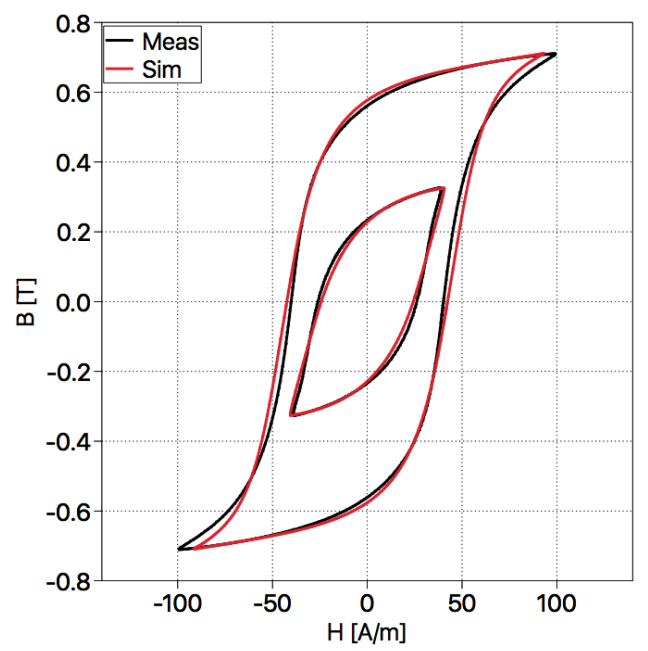

(a)

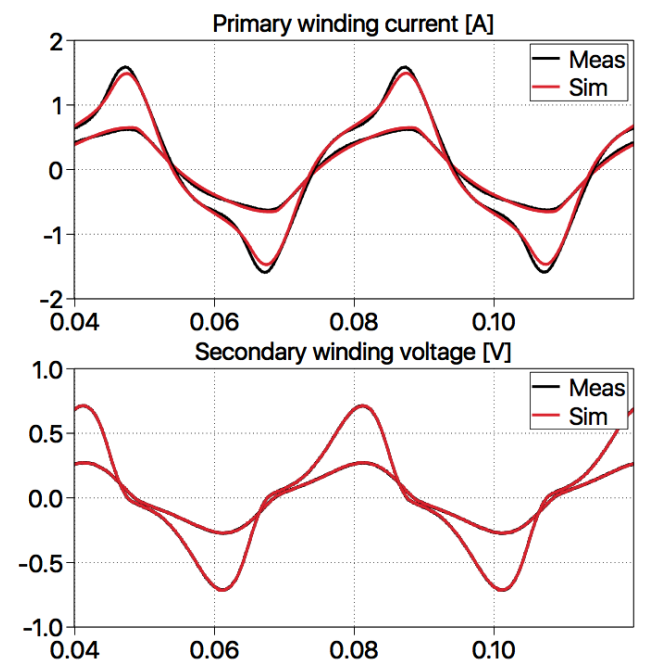

(b)

Fig. 7: Comparison between measurement and simulation under $25 \mathrm{~Hz}$ excitation with different amplitudes (a) B-H loops; (b) Time domain waveforms.

calculated using the equations (1), (2) and (4). Please note that the parameters determined from this stage will remain the same for the verification.

The simulation model established in the system level simulation software PLECS includes the core sample using the proposed magnetic circuit as shown in Fig. 6b. A controlled voltage source is fed by measured secondary voltage value multiplied by a factor $N_{1} / N_{2}$, which is equal to the excitation voltage on the primary winding with the voltage drop on the winding- and cir-

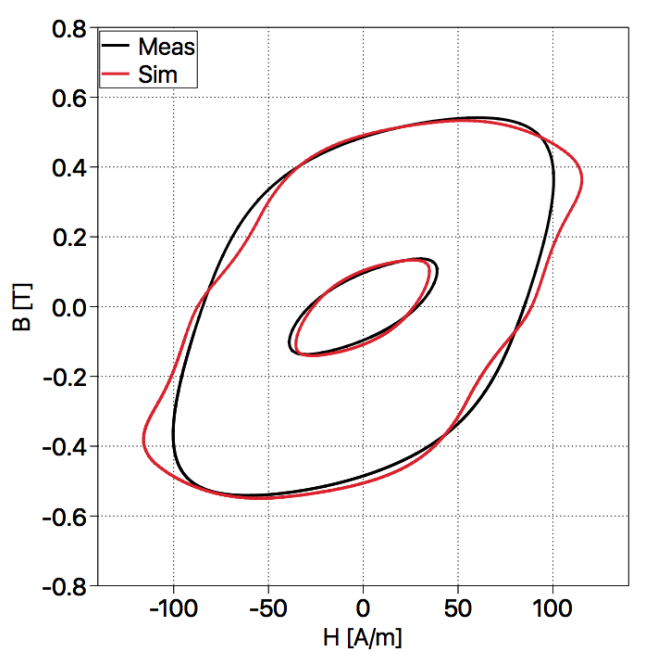

(a)
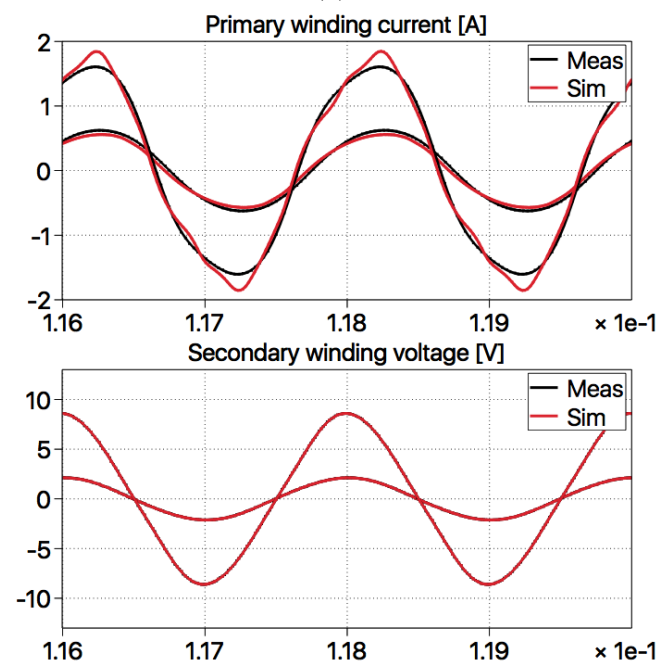

(b)

Fig. 8: Comparison between measurement and simulation under $500 \mathrm{~Hz}$ excitation with different amplitudes (a) B-H loops; (b) Time domain waveforms.

cuit resistance excluded. All components are configured to be same as the test bench hardware in each of the following schemes.

The verification is firstly carried out with low frequency excitation of $25 \mathrm{~Hz}$. In Fig. 7 the simulated B-H loops of different amplitudes as well as the corresponding primary winding current and secondary winding voltage are compared to the measurement. The simulation model is able to accurately reproduce the distorted time domain waveform which reflects the influence of the ma- 


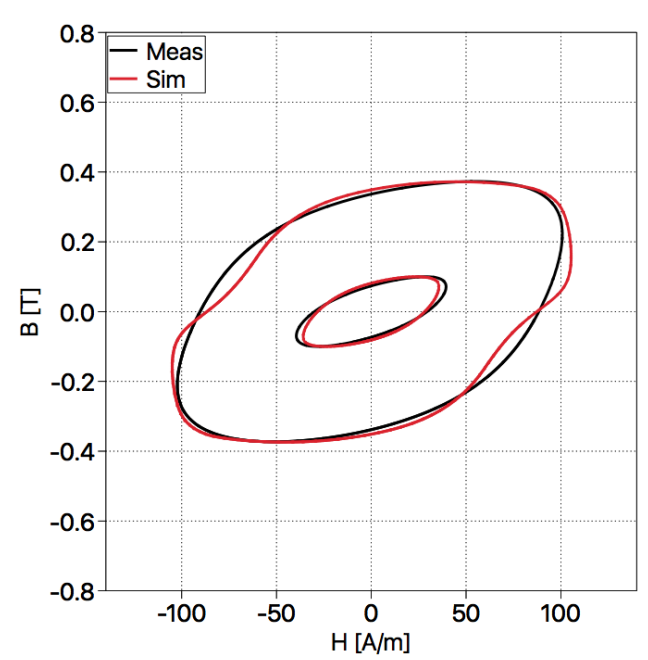

(a)
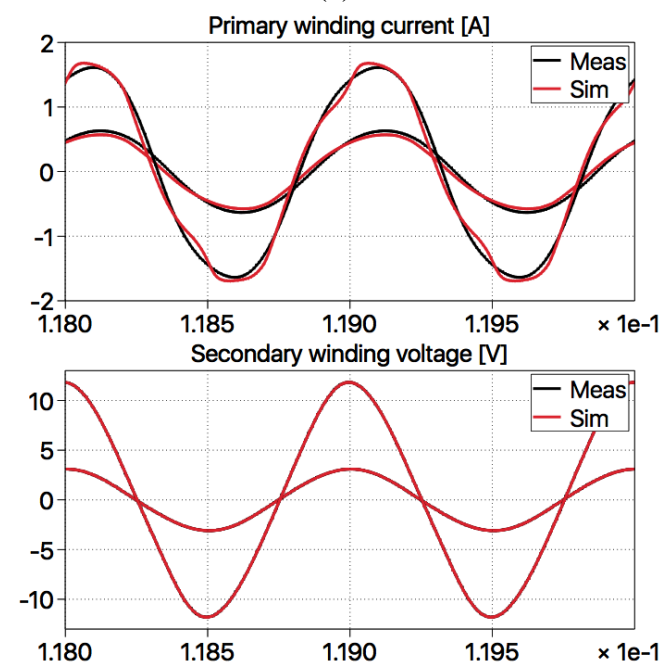

(b)

Fig. 9: Comparison between measurement and simulation under $1 \mathrm{kHz}$ excitation with different amplitudes (a) B-H loops; (b) Time domain waveforms.

terial's hysteresis nonlinearity. The per-cycle energy loss is calculated via integrating the product of the primary current and secondary voltage for one AC period, the maximum error of the simulation results turns out to be 3\%. Then in Fig. 8 and Fig. 9 the simulation results under $500 \mathrm{~Hz}$ and $1 \mathrm{kHz}$ excitation are compared with the measurement. With increased excitation frequency, the eddy current contributes even more to the per-cycle energy loss, in comparison to the frequency-independent hysteresis loss. Again good match is obtained in both B-
$\mathrm{H}$ characteristic and time domain waveforms, the error of the per-cycle energy loss is $4.1 \%$ at $500 \mathrm{~Hz}$ and $1.7 \%$ at $1 k H z$. The decreased error at $1 k H z$ can be ascribed to the fact that with increased frequency the magnetic flux is pushed towards the surface of the laminate, thanks to the nonuniform division of sub-laminates, the geometry near the surface is more precisely described.

After the model is established and parameterised based on the sample core, the proposed magnetic circuit can be configured into different geometry and taken as a building block to compose iron cores with complex structures. A rudimentary transformer prototype is constructed as shown in Fig. 10a, with three-limb iron core made of laminates having the same material and thickness as the square core sample. The number of the stacked laminates here is 20. Two windings with turns number $N_{1}=N_{2}=$ 20 are installed on the middle limb. The magnetic circuit model is composed of three ladder structures (Fig. 6b) in parallel connection, which represent the middle- and

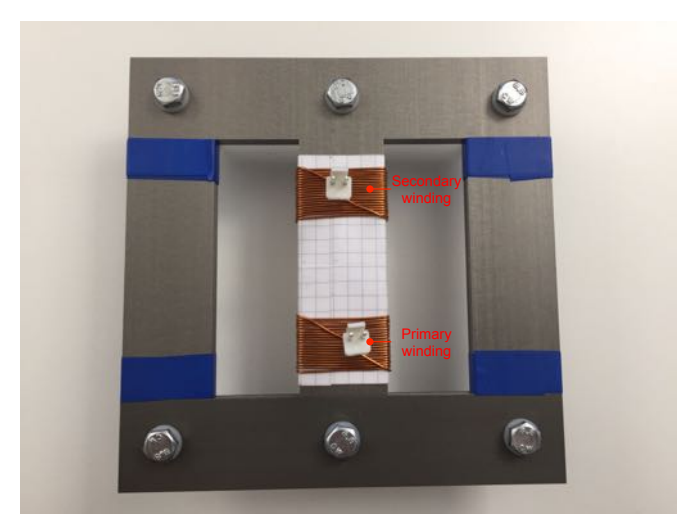

(a)

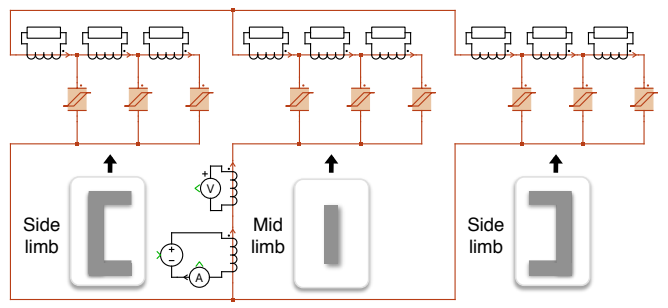

(b)

Fig. 10: Three limb transformer prototype (a) Hardware structure; (b) Magnetic circuit model using proposed approach. 


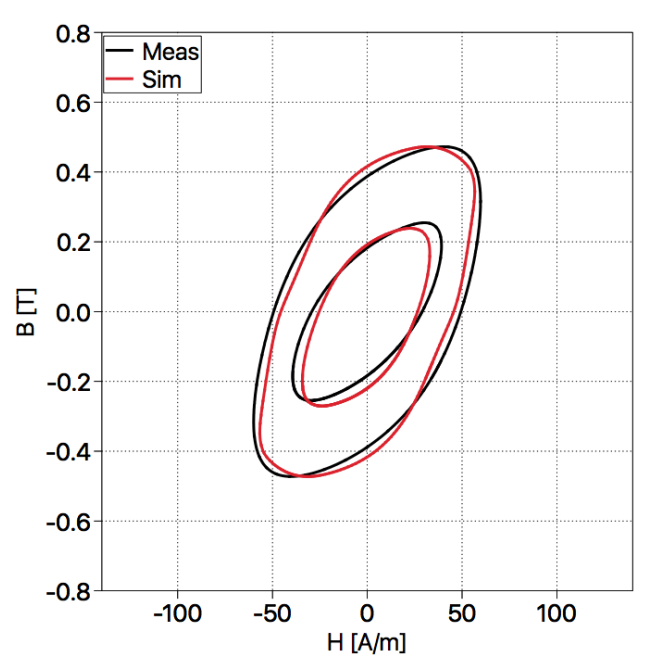

(a)
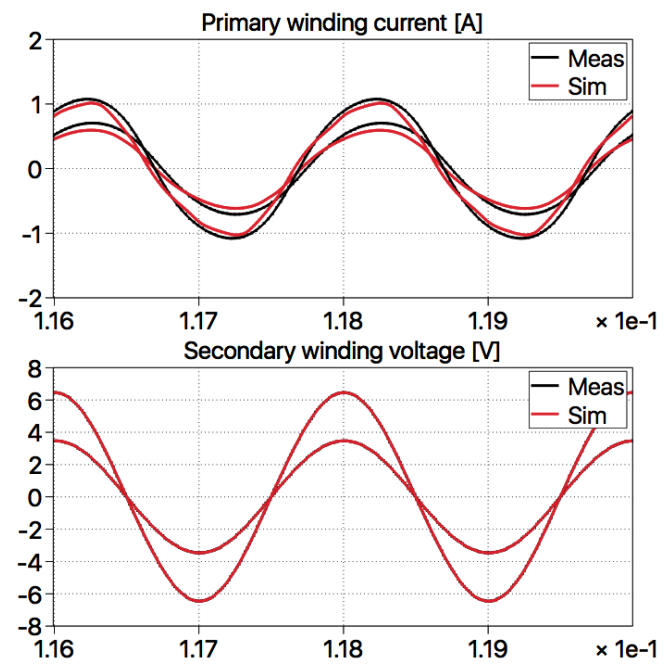

(b)

Fig. 11: Comparison between measurement and simulation of the three limb transformer prototype under $500 \mathrm{~Hz}$ excitation with different amplitudes (a) B-H loops; (b) Time domain waveforms.

side limbs, respectively, as depicted in Fig. 10b. The two winding components are placed on the corresponding locations. In comparison to the model presented in Fig. $6 \mathrm{~b}$, only the geometrical parameters $l$ and $w$ as well as the stacking number $K$ need to be adapted. Please note that the material related parameters for calculating the nonlinear permeabilities $\mu^{i r r}$ and $\mu_{\text {rev }}$, which has been identified in a previous stage, remain the same.

For verification, the primary winding is supplied by
$500 \mathrm{~Hz}$ sinusoidal voltage, while the secondary winding is left open. Similar to the configuration of the magnetic circuit model in Fig. 6b, the measured secondary winding voltage is multiplied by the factor $N_{1} / N_{2}$ and provided to the controlled voltage source as reference on the primary winding. In Fig. 11 the B-H loops and time domain waveforms are compared between simulation and measurement. After adapting the geometry while making use of the same material representation, the model of the three limb is still able to reproduce the measurement with good accuracy. The maximum error of the per-cycle energy loss is $6.2 \%$.

\section{CONCLusion}

This work has proposed a core model for time domain system-level simulation using permeance-capacitance based magnetic circuit, which captures the frequencyindependent magnetic hysteresis of the material together with the frequency-dependent eddy current effect. Parameters of the model are identified via experimentally measured low frequency limiting loop as well as geometrical parameters. The fidelity of the model has been verified via experimental tests under excitation of different amplitude and frequencies on a sample core. The magnetic circuit can be easily extended to represent complex core geometries, which has been verified in terms of time domain waveform as well as per-cycle energy loss as well.

\section{ACKNOWLEDGMENT}

This project has been supported in the frame of the ECPE Joint Research Programme.

\section{REFERENCES}

[1] J. A. Martinez and B. A. Mork, "Transformer modeling for lowand mid-frequency transients - a review," in IEEE Transactions on Power Delivery, vol. 20, no. 2, 2005, pp. 1625-1632.

[2] F. de Leon and A. Semlyen, "Time domain modeling of eddy currrent effects for transformer transients," in IEEE Transactions on Power Delivery, vol. 8, no. 1, 1993, pp. 271-280.

[3] E. J. Tarasiewicz, A. S. Morcheb, A. Narang, and E. P. Dick, "Frequency dependent current models for nonlinear iron cores," in IEEE Transactions on Power Systems, vol. 8, no. 2, 1993, pp. 588-596. 
[4] A. Davoudi, P. L. Chapman, J. Jatskevich, and A. Khaligh, "Reduced-order modeling of high-fidelity mangetic equivalent circuits," in IEEE Transactions on Power Electronics, vol. 24, no. 12,2009 , pp. 2847-2855.

[5] A. Davoudi, P. L. Chapman, J. Jatskevich, and H. Behjati, "Reduced-order modeling of multi-winding power electronic magnetic components," in IEEE Transactions on Power Electronics, vol. 27, no. 5, 2012, pp. 2220-2226.

[6] P. Holmberg and A. Bergqvist, "Modelling eddy current and hysteresis in a trasnformer laminate," in IEEE Transactions on Magnetics, vol. 33, no. 2, 1997, pp. 1306-1309.

[7] J. G. Zhu, R. Hui, and V. S. Ramsden, "A dynamic equivalent circuit model for solid magnetic cores for high switching frequency operations," in IEEE Transactions on Power Electronics, vol. 10, 1995, pp. 791-795.

[8] D. Hamill, "Lumped equivalent circuits of magnetic components: the gyrator-capacitor approach," in IEEE Transactions on Power Electronics, vol. 8, 1994, pp. 97-103.

[9] L. Yan and B. Lehman, "A capacitor modeling method for integrated magnetic components in $\mathrm{dc} / \mathrm{dc}$ converters," in IEEE Transactions on Power Electronics, vol. 20, no. 5, 2005, pp. 987-996.

[10] J. Allmeling, W. Hammer, and J. Schönberger, "Transient simulation of magnetic circuits using the permeance-capacitance analogy," in Control and Modeling for Power Electronics (COMPEL), IEEE 13th Workshop on, 2012.

[11] M. Luo, D. Dujic, and J. Allmeling, "Leakage flux modeling of multi-winding transformers for system-level simulations," in IEEE Transactions on Power Electronics, 2017, Early Access.

[12] M. Luo and D. Dujic and J. Allmeling, "Modelling hysteresis of soft core materials using permeance-capacitance analogy for transient circuit simulations," in European Conference on Power Electronics and Applications 2017 (EPE, ECCE Europe), 2017, pp. P.1-P.10.

[13] P. G. Blanken, "A lumped winding model for use in transformer models for circuit simulation," in IEEE Transactions on Power Electronics, vol. 16, no. 3, 2001, pp. 445-460.

[14] B. Azzerboni, E. Cardelli, G. Finocchio, and F. L. Foresta, "Remarks about preisach function approximation using lorentzian function and its identification for nonoriented steels," in IEEE Transactions on Magnetics, vol. 39, no. 5, 2003, pp. 3028-3030.

[15] C. Perez-Rojas, "Fitting saturation and hysteresis via arctangent funcitons," in IEEE Power Engineering Review, vol. 20, no. 1, 2000, pp. 55-57.

[16] D. Tan, J. L. Vollin, and S. M. Cuk, "A practical approach for magnetic core-loss characterization," in IEEE Transactions on Power Electronics, vol. 10, no. 2, 1995, pp. 124-130. 